\title{
Socio-demographic correlates of unhealthy lifestyle in Ethiopia: a secondary analysis of a national survey
}

Yalemzewod Assefa Gelaw ( $\square$ yalassefa@gmail.com )

Curtin University

\section{Digsu N. Koye}

The University of Melbourne

Kefyalew Addis Alene

Curtin University

\section{Kedir Yimam Ahmed}

Translational Health Research Institute, Western Sydney University-Campbelltown Campus

\section{Yibeltal Assefa}

The University of Queensland

\section{Daniel Asfaw Erku}

Griffith University

\section{Henok Getachew Tegegn}

University of New England

Azeb Gebresilassie Tesema

University of New South Wales

Berihun Megabiaw Zeleke

Monash University

Yohannes Adama Melaku

Flinders University

\section{Research Article}

Keywords:

Posted Date: March 7th, 2022

DOI: https://doi.org/10.21203/rs.3.rs-1401928/v1

License: (c) (1) This work is licensed under a Creative Commons Attribution 4.0 International License.

Read Full License 


\section{Abstract}

This study aimed to assess socio-demographic correlates of unhealthy lifestyles among adolescents and adults in Ethiopia. A population-based national cross-sectional survey using the WHO NCD STEPS instruments was conducted. Data were collected in 2015 , from a total of 9,800 participants aged between 15 and 69 years. Unhealthy lifestyle scores ( 0 [most healthy] to 5 [most unhealthy]) were determined based on diet (daily fruit and vegetable consumption), smoking status, overweight/obesity, alcohol intake, and physical activity. A co-occurrence of 3 or more unhealthy lifestyles was considered as an unhealthy lifestyle. The study found that $98.2 \%$ of participants had low consumptions of fruit and vegetables, $5.4 \%$ smoked tobacco, $15.0 \%$ consumed excessive alcohol, $66.0 \%$ had inadequate physical activity and $2.3 \%$ were obese. One in eight $(13 \%)$ participants were having three or more unhealthy lifestyles. We found that male sex, urban residence, older age, being married or living in common-law, and higher income were associated with unhealthy lifestyles. Participants with higher educational status had lower odds of unhealthy lifestyles. Socio-demographic characteristics such as sex, age, marital status, residence, income, and educational status were correlated with individuals' lifestyles. Tailored interventions that target specific socio-demographic groups are required to address the increasing burden of unhealthy lifestyles in Ethiopia.

\section{Introduction}

Cardiovascular diseases, cancer, chronic respiratory diseases, and diabetes are the most common type of NCDs accounting for $80 \%$ of premature NCD-related deaths (1) and contributing to more than 41 million deaths due to NCD-related causes every year $(2,3)$. The burden of NCDs has increased by almost $14 \%$ since 2005 and has created major concerns for society and governments. Low-and middle-income countries (LMICs) are highly affected, accounting for almost $77 \%$ of all deaths and $85 \%$ of premature deaths (aged 30-69 years) (2).

The burden of NCDs poses social, economic, and health inequality disproportionately affecting poor communities. The adverse consequences of NCDs are more pronounced in LMICs (4) due to the double burden of infectious diseases and weak healthcare systems $(5,6)$. Tobacco use, harmful use of alcohol, physical inactivity, and unhealthy diet are the four most common modifiable lifestyle risk factors that have been shown to increase the risk of NCDs (7). Each year, tobacco accounts for over 7.2 million deaths, excess salt or sodium intake accounts for 4.1 million deaths, 3.3 million deaths attributed to harmful use of alcohol, and 1.6 million deaths attributed to insufficient physical activity (8). Metabolic risk factors including overweight and obesity elevated blood pressure and raised blood glucose are associated with these modifiable lifestyle risk factors and contribute to the risk of developing NCDs $(7,8)$. Consequently, global efforts to control NCD focuses on addressing these lifestyle factors (2).

The sustainable development goal (SDG) target 3.4 aims to reduce NCD-related premature death by onethird by 2030 (9). In line with this goal, Ethiopia set targets to reduce NCD-related premature deaths by $12.5 \%$ (10). Though deaths due to NCDs were reduced by $37 \%$ between 1990 and 2015 in Ethiopia, 
knowledge regarding the burden of unhealthy lifestyle factors and their association with sociodemographic and metabolic factors is limited (11). This reflects that the national strategies require full implementation of interventions and need to prioritize people with unhealthy lifestyle factors associated with NCDs.

The evidence for the benefit of healthy behaviours on reducing NCDs is well established (12-14). Multiple lifestyle risk factors are more likely to have a stronger association with NCDs than a single lifestyle-related factor. Hence, it would be of special interest to combine all the major lifestyle risk factors and determine an individual's overall unhealthy lifestyle so that individuals at significant risk for NCDs could be targeted and included in the intervention programs (15). Understanding socio-demographic factors, such as income inequality, with unhealthy lifestyles, has recently attracted more attention (1618). Targeting socio-demographic groups with multiple unhealthy lifestyles could be a cost-effective strategy to prioritize NCD prevention interventions $(19,20)$. However, no study, to our knowledge, has shown the socio-demographic correlates of multiple unhealthy lifestyles in Ethiopia. Therefore, this study aimed at determining the prevalence of unhealthy lifestyles and their association with socio-demographic groups. In addition, we also assessed the association of unhealthy lifestyles with diabetes and hypertension.

\section{Methods}

\section{Data source}

Individual-level data collected from a community-based cross-sectional survey were used. The survey was collected in 2015 by the Ethiopian Public Health Institute in collaboration with the Federal Ethiopian Ministry of Health (FMOH) and World Health Organization (WHO) using the NCD STEPS instrument (21). The STEPS survey methods are described in detail elsewhere (22). Briefly, the survey was conducted among 9, 800 adolescents and adults aged between 15 and 69 years. The survey contains information about the socio-demographic and behavioural characteristics (STEP I); physical measurements for blood pressure, overweight, and obesity were calculated (STEP II); and included biochemical measurement for diabetes, raised blood glucose, and abnormal lipid level (STEP III).

\section{Sociodemographic characteristics}

Sociodemographic data such as age (15-29, 30-44, 45-59, and 60-69 years), sex, residence (urban or rural), education (no formal schooling, primary school completed, secondary school completed, and college/University completed), marital status (single, married, and common-law), and income $(\leq 12,000$, $12,000-23,299, \geq 23,300$ Birr) were assessed. Participants were recruited from all administrative regions of Ethiopia.

\section{Unhealthy lifestyle score}

Based on previous studies (23), insufficient physical activity, tobacco use, excessive intake of alcohol, and inadequate serving of fruit and vegetable intake, and overweight/obesity were used to construct 
unhealthy lifestyle scores as an outcome variable.

\section{Insufficient physical activity}

Physical activity was assessed based on the total time spent on physical activity per day at work, including transport and recreational settings. It was measured using the metabolic equivalent time (MET) in minutes per week spent in physical activity. According to the WHO recommendation on physical activity, performing an equivalent combination of moderate- and vigorous-intensity physical activity below 600 MET-minutes per week was considered as insufficient physical activity (24).

\section{Excessive alcohol intake}

Alcohol consumption was measured in terms of current and previous drinking (i.e., ever or within 12 months of the interview period) using the concept of a standard drink i.e., any drink containing about $10 \mathrm{~g}$ of pure alcohol. Men who reported four or more standard units per day and women who reported three and more standard units per day "ever" or within the last 12 months were classified as having excessive use of alcohol (25).

\section{Current smoking}

Tobacco use was assessed in terms of current and previous smoking status, duration of smoking, the quantity of tobacco use, smokeless tobacco use, and exposure to second-hand smoking. Respondents who replied, 'Yes' for the question "do you smoke cigarettes (any tobacco product)?" were categorized as "current tobacco users".

\section{Dietary intake}

fruit and vegetable intake were used as a surrogate variable for overall dietary quality as there was no comprehensive data on dietary intake. Consumption of fruit and vegetables was assessed in terms of the number of servings, with a serving being equal to $400 \mathrm{~g}$ (26). Participants who reported consumptions of less than five servings of fruit or vegetables per day during the last 30 days of the interview were considered as having a suboptimal diet (27).

\section{Metabolic factors}

\section{Overweight/obesity}

a respondent's height $(\mathrm{cm})$ and weight $(\mathrm{kg})$ measurements were taken to calculate body mass index (BMI). BMI was calculated as the respondent's weight in kilograms divided by the square of the respondent's height in meters $(\mathrm{kg} / \mathrm{m} 2)$. Participants with BMI $25-29.9$ and $\geq 30 \mathrm{~kg} / \mathrm{m} 2$ were classified as overweight and obese, respectively.

The unhealthy lifestyle scores for each participant were calculated as the sum of the five indicators and possible scores ranged from 0 (lowest unhealthy score) to 5 (highest unhealthy score). The highest 
scores represent the extent of co-occurrence of risk factors. We defined an unhealthy lifestyle as cooccurrence of 3 or more risk factors. Since most (around three in four) of the respondents were from the rural area of Ethiopia, many of the participants could have at least two unhealthy lifestyle risk factors due to the following reasons: (i) home-made beer-like traditional alcohol (called 'Tela) intake is common in rural areas and (ii) knowledge of nutrition and dietary diversity are low in rural residents (28).

Diabetes: Blood glucose measurements were taken to assess diabetes. Diabetes was defined as fasting plasma glucose value: $>=7.0 \mathrm{mmol} / \mathrm{L}(126 \mathrm{mg} / \mathrm{dl})$ or currently taking medication/s for diabetes.

\section{High blood pressure}

Both systolic (SBP) and diastolic (DBP) blood pressure were measured three times and the mean values were taken for this analysis. High blood pressure was defined as SBP $>=140 \mathrm{mmHg}$ and/or DBP $>=90$, or currently taking medication for high blood pressure.

\section{Statistical analysis}

Demographic characteristics were summarised using proportion (categorical variables), mean and SD (continuous and symmetrically distributed data), or median and interquartile ranges (25th and 75th percentiles) for (continuous and asymmetric data). Bivariable and multivariable logistic regression models were used to determine the association of socio-demographic factors with unhealthy lifestyle using crude and adjusted odds ratio (OR) and 95\% confidence interval $(\mathrm{Cl})$. A p-value $<0.05$ is considered statistically significant for the association between socio-demographic factors and co-occurrence of unhealthy NCD lifestyle risk factors. Data management and analysis were performed using Stata version 16 (StataCorp LLC, College Station, TX) and R (version 3.6.3, R Foundation for Statistical Computing, Vienna, Austria). All methods were carried out in accordance with the Strengthening the Reporting of Observational studies in Epidemiology (STROBE) guidelines (29).

\section{Results}

A total of 9,800 participants were included in the analysis with a response rate of $95.5 \%$. Three in five participants were females (59.4\%), 78.8\% did not attend formal schooling and $72.6 \%$ were rural residents. The median age of the study participants was 32 years (25th and 75th percentile: $25,44 \mathrm{y}$ ). Two-thirds of the participants $(66.0 \%)$ had insufficient physical activity per week, $15.3 \%$ were classified as they drank excess alcohol per day based on their response and $5.4 \%$ of participants were current smokers. Almost all had inadequate fruit and vegetable intake (98.2\%) and $10 \%$ were overweight/obese. There were significant differences in the prevalence of almost all unhealthy behaviours across sociodemographic characteristics (Table 1). 
Table 1

Socio-demographic characteristics of the study participant and unhealthy lifestyle factors in Ethiopia

\section{Unhealthy lifestyle factors}

Variables

7,113

(72.7)

$\operatorname{Sex}(N=9,800)$

Female

Age groups in

years $(N=9,800)$

15-29

$30-44$

45-59

$60-69$

Marital status (N

=9,799)

Single

Married

Insufficient
physical
activity

Total $\quad 6,012(66.0)$

sample

n (\%)
Excessive alcohol intake

1,441

(15.3)
Current smoking

533

(5.4)
Low fruit \& vegetable intake

9,524 (98.2) $938(10.1)$
Overweight and Obesity 938 (10.1)

\begin{tabular}{lllllll}
$\begin{array}{l}\text { Residence }(N= \\
9,800)\end{array}$ & & $p=0.023$ & $p=0.01$ & $p=0.00$ & $p=0.76$ & $p<0.00$ \\
Rural & $\begin{array}{l}7,113 \\
(72.7)\end{array}$ & $4,428(65.3)$ & $\begin{array}{l}1,099 \\
(16.0)\end{array}$ & $\begin{array}{l}421 \\
(6.0)\end{array}$ & $6,923(98.0)$ & $322(4.9)$ \\
& & & & & \\
\hline
\end{tabular}

\begin{tabular}{lllllll}
$\begin{array}{l}\text { Residence }(N= \\
9,800)\end{array}$ & & $p=0.023$ & $p=0.01$ & $p=0.00$ & $p=0.76$ & $p<0.00$ \\
Rural & $\begin{array}{l}7,113 \\
(72.7)\end{array}$ & $4,428(65.3)$ & $\begin{array}{l}1,099 \\
(16.0)\end{array}$ & $\begin{array}{l}421 \\
(6.0)\end{array}$ & $6,923(98.0)$ & $322(4.9)$ \\
& & & & & \\
\hline
\end{tabular}

\begin{tabular}{lllllll}
$\begin{array}{l}\text { Residence }(N= \\
9,800)\end{array}$ & & $p=0.023$ & $p=0.01$ & $p=0.00$ & $p=0.76$ & $p<0.00$ \\
Rural & $\begin{array}{l}7,113 \\
(72.7)\end{array}$ & $4,428(65.3)$ & $\begin{array}{l}1,099 \\
(16.0)\end{array}$ & $\begin{array}{l}421 \\
(6.0)\end{array}$ & $6,923(98.0)$ & $322(4.9)$ \\
& & & & & \\
\hline
\end{tabular}

$\begin{array}{lllllll}\begin{array}{l}\text { Residence }(N= \\ 9,800)\end{array} & & p=0.023 & p=0.01 & p=0.00 & p=0.76 & p<0.00 \\ \text { Rural } & \begin{array}{lllll}7,113 \\ (72.7)\end{array} & 4,428(65.3) & \begin{array}{l}1,099 \\ (16.0)\end{array} & \begin{array}{l}421 \\ (6.0)\end{array} & 6,923(98.0) & 322(4.9) \\ & & & & \end{array}$

$p=0.76$




\section{Unhealthy lifestyle factors}

\begin{tabular}{|c|c|c|c|c|c|c|}
\hline common-law* & $\begin{array}{l}1,501 \\
(15.3)\end{array}$ & $903(67.8)$ & $190(13.3)$ & $64(4.3)$ & $1,449(98.4)$ & $196(13.5)$ \\
\hline $\begin{array}{l}\text { Highest level of } \\
\text { education }\end{array}$ & & $p<0.00$ & $p=0.07$ & $p=0.65$ & $p=0.00$ & $p<0.00$ \\
\hline \multicolumn{7}{|l|}{$(N=9,800)$} \\
\hline $\begin{array}{l}\text { No formal } \\
\text { Schooling }\end{array}$ & $\begin{array}{l}7,661 \\
(78.2)\end{array}$ & 4,808 (67.0) & $\begin{array}{l}1,147 \\
(15.5)\end{array}$ & $\begin{array}{l}406 \\
(5.3)\end{array}$ & $7,641(98.5)$ & 604 (8.3) \\
\hline $\begin{array}{l}\text { Primary school } \\
\text { completed }\end{array}$ & $\begin{array}{l}975 \\
(10.0)\end{array}$ & $579(65.0)$ & $124(13.6)$ & $59(6.1)$ & $941(97.3)$ & $104(11.3)$ \\
\hline $\begin{array}{l}\text { Secondary school } \\
\text { completed }\end{array}$ & $\begin{array}{l}653 \\
(6.7)\end{array}$ & $372(63.0)$ & $82(13.6)$ & $40(6.1)$ & $631(97.8)$ & $120(19.0)$ \\
\hline $\begin{array}{l}\text { College/University } \\
\text { completed* }\end{array}$ & $\begin{array}{l}511 \\
(5.2)\end{array}$ & $253(55.0)$ & $88(18.3)$ & $28(5.5)$ & $491(97.0)$ & $110(22.8)$ \\
\hline $\begin{array}{l}\text { Annual income } \\
(N=6,636)\end{array}$ & & $p<0.00$ & $p<0.00$ & $p=0.65$ & $p=0.00$ & $p<0.00$ \\
\hline$<=12,000$ Birr & $\begin{array}{l}4,494 \\
(67.7)\end{array}$ & 2,978 (71.2) & $525(12.1)$ & $\begin{array}{l}232 \\
(5.2)\end{array}$ & $4,320(97.4)$ & $399(9.4)$ \\
\hline 12,000 to 23,299 & $\begin{array}{l}1,017 \\
(15.3)\end{array}$ & $634(65.6)$ & $214(21.8)$ & $48(4.7)$ & $1,003(99.2)$ & $112(11.5)$ \\
\hline $23,300^{+}$ & $\begin{array}{l}1,125 \\
(17.0)\end{array}$ & $661(64.7)$ & $184(17.3)$ & $63(5.6)$ & $1,084(97.2)$ & $213(20.1)$ \\
\hline $\begin{array}{l}\text { Regions }(N= \\
9,800)\end{array}$ & & $p<0.00$ & $p<0.00$ & $p<0.00$ & - & - \\
\hline Addis Ababa & $\begin{array}{l}815 \\
(6.7)\end{array}$ & $464(75.2)$ & $105(15.0)$ & $28(3.4)$ & $771(98.3)$ & $259(33.0)$ \\
\hline Afar & $\begin{array}{l}384 \\
(2.1)\end{array}$ & $284(74.3)$ & $3(0.8)$ & $36(9.4)$ & $383(99.7)$ & $20(5.8)$ \\
\hline Amhara & $\begin{array}{l}1,867 \\
(10.6)\end{array}$ & $1,064(59.5)$ & $479(26.8)$ & $34(1.8)$ & $1,861(99.9)$ & $96(5.3)$ \\
\hline
\end{tabular}

Postgraduate completed $(n=12) ; *$ common-law (Separated $=386$, Divorced $=402$, widowed $=669$, cohabitating $=41$, Refused $=3$ ).

^SBP > = 140/DBP > = 90; diabetes (Yes: FBG > = 110)

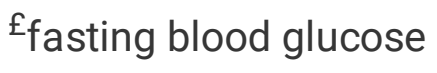

SBP: systolic blood pressure; DBP: diastolic blood pressure 


\begin{tabular}{|c|c|c|c|c|c|c|}
\hline \multirow[b]{2}{*}{ B. Gumuz } & \multicolumn{6}{|c|}{ Unhealthy lifestyle factors } \\
\hline & $\begin{array}{l}384 \\
(2.0)\end{array}$ & $263(71.1)$ & $105(28.1)$ & $20(5.2)$ & $379(99.2)$ & $25(6.9)$ \\
\hline Dire Dawa & $\begin{array}{l}257 \\
(1.6)\end{array}$ & $164(73.7)$ & $7(2.7)$ & $\begin{array}{l}32 \\
(12.5)\end{array}$ & $254(99.2)$ & $29(12.3)$ \\
\hline Gambela & $\begin{array}{l}295 \\
(3.0)\end{array}$ & $103(36.4)$ & $75(26)$ & $65(22)$ & $290(99.3)$ & $27(9.4)$ \\
\hline Harari & $\begin{array}{l}214 \\
(2.2)\end{array}$ & $84(70)$ & $0(0)$ & $\begin{array}{l}35 \\
(16.4)\end{array}$ & $198(100)$ & $25(12.2)$ \\
\hline Oromia & $\begin{array}{l}2,308 \\
(23.5)\end{array}$ & $1,561(70)$ & $233(10.5)$ & $\begin{array}{l}130 \\
(5.6)\end{array}$ & 2,285 (99.3) & $191(8.7)$ \\
\hline SNNPR & $\begin{array}{l}1,706 \\
(17.4)\end{array}$ & $1,079(66)$ & $196(11.8)$ & $62(3.6)$ & $1,552(92.7)$ & $137(8.6)$ \\
\hline Somali & $\begin{array}{l}615 \\
(6.3)\end{array}$ & $255(47.1)$ & $14(2.3)$ & $\begin{array}{l}89 \\
(14.5)\end{array}$ & $601(98.4)$ & $84(14.6)$ \\
\hline Tigray & $\begin{array}{l}955 \\
(9.7)\end{array}$ & $691(75.8)$ & $224(24.5)$ & $2(0.2)$ & $950(99.8)$ & $45(5.0)$ \\
\hline $\begin{array}{l}\text { Diabetes }(N= \\
8,790)\end{array}$ & & $p=0.75$ & $p=0.05$ & $\begin{array}{l}p= \\
0.048\end{array}$ & - & $p<0.00$ \\
\hline Yes & $\begin{array}{l}244 \\
(2.8)\end{array}$ & $138(67.0)$ & $24(10.4)$ & $20(8.2)$ & 235 (98.3) & $62(25.9)$ \\
\hline $\begin{array}{l}\text { Hypertension }(N= \\
9,675)\end{array}$ & & $p=0.91$ & $p=0.00$ & $p=0.75$ & $p=0.95$ & $P<0.00$ \\
\hline $\begin{array}{l}\mathrm{SBP}>=140 \text { or } \\
\mathrm{DBP}>=90\end{array}$ & $\begin{array}{l}1,871 \\
(19.3)\end{array}$ & $1,115(66.1)$ & $315(17.8)$ & $99(5.3)$ & $1,818(98.3)$ & $383(20.7)$ \\
\hline \multicolumn{7}{|c|}{$\begin{array}{l}\text { Postgraduate completed }(n=12) ; * \text { common-law (Separated }=386 \text {, Divorced }=402 \text {, widowed }=669 \text {, } \\
\text { cohabitating }=41 \text {, Refused }=3 \text { ). }\end{array}$} \\
\hline \multicolumn{7}{|c|}{${ }^{\wedge} \mathrm{SBP}>=140 / \mathrm{DBP}>=90$; diabetes $($ Yes: FBG > = 110) } \\
\hline \multicolumn{7}{|c|}{$\varepsilon_{\text {fasting blood glucose }}$} \\
\hline SBP: systolic blood & essure & P: diastolic & d pressur & & & \\
\hline
\end{tabular}

\section{Co-occurrences of unhealthy lifestyles}

Table 2 shows sociodemographic characteristics across unhealthy lifestyle scores. The median unhealthy lifestyle score was three, only three participants had five risk factors and one in six participants $(16.7 \%)$ had three or more unhealthy lifestyle factors. There were significant differences in unhealthy lifestyle scores across sociodemographic categories. A higher unhealthy lifestyle score was common in 
older age rural participants (Fig. 1). In most regional administration areas, participants had a least 2 unhealthy lifestyle factors (Fig. 2). 
Table 2

The numbers of unhealthy lifestyles and socio-demographic characteristics of the study participants in Ethiopia $(\mathrm{N}=9,796)$.

\begin{tabular}{|c|c|c|c|c|c|c|}
\hline & & \multicolumn{5}{|c|}{ Number of risk factors } \\
\hline & & None & One & Two & Three & Four \\
\hline Variables & $\mathrm{n}(\%)$ & $\begin{array}{l}69 \\
(0.8)\end{array}$ & $\begin{array}{l}2,806 \\
(32.0)\end{array}$ & $\begin{array}{l}5274 \\
(55.0)\end{array}$ & $\begin{array}{l}1,501 \\
(12.0)\end{array}$ & $\begin{array}{l}147 \\
(0.7)\end{array}$ \\
\hline \multicolumn{7}{|l|}{ Residence $(N=9,797)$} \\
\hline Rural & $7,111(72.7)$ & $\begin{array}{l}44 \\
(0.6)\end{array}$ & $2,019(3)$ & $\begin{array}{l}3,801 \\
(55.1)\end{array}$ & $\begin{array}{l}953 \\
(13.8)\end{array}$ & $\begin{array}{l}85 \\
(1.2)\end{array}$ \\
\hline \multicolumn{7}{|l|}{$\operatorname{Sex}(N=9,797)$} \\
\hline Female & $\begin{array}{l}5,821 \\
(59.4)\end{array}$ & $\begin{array}{l}49 \\
(0.8)\end{array}$ & $\begin{array}{l}1,788 \\
(30.7)\end{array}$ & $\begin{array}{l}3,273 \\
(56.2)\end{array}$ & $\begin{array}{l}659 \\
(11.3)\end{array}$ & $\begin{array}{l}52 \\
(0.9)\end{array}$ \\
\hline \multicolumn{7}{|c|}{ Age groups in year $(N=9,797)$} \\
\hline $15-29$ years & $\begin{array}{l}3,959 \\
(40.4)\end{array}$ & $\begin{array}{l}24 \\
(0.6)\end{array}$ & $\begin{array}{l}1,272 \\
(32.1)\end{array}$ & $2,30(564)$ & $392(9.9)$ & $\begin{array}{l}39 \\
(1.0)\end{array}$ \\
\hline $30-44$ years & $\begin{array}{l}3,499 \\
(35.7)\end{array}$ & $\begin{array}{l}24 \\
(0.7)\end{array}$ & $\begin{array}{l}940 \\
(26.9)\end{array}$ & $\begin{array}{l}1,865 \\
(53.3)\end{array}$ & $\begin{array}{l}614 \\
(17.6)\end{array}$ & $\begin{array}{l}55 \\
(1.6)\end{array}$ \\
\hline $45-59$ years & $\begin{array}{l}1,690 \\
(17.2)\end{array}$ & $\begin{array}{l}14 \\
(0.8)\end{array}$ & $\begin{array}{l}413 \\
(24.4)\end{array}$ & $\begin{array}{l}846 \\
(50.1)\end{array}$ & $\begin{array}{l}370 \\
(21.9)\end{array}$ & $\begin{array}{l}47 \\
(2.8)\end{array}$ \\
\hline $60-69$ years & $652(6.7)$ & $\begin{array}{l}7 \\
(1.1)\end{array}$ & $18127.8)$ & $\begin{array}{l}333 \\
(51.1)\end{array}$ & $\begin{array}{l}125 \\
(19.2)\end{array}$ & $6(0.9)$ \\
\hline \multicolumn{7}{|c|}{ Marital status $(N=9,799)$} \\
\hline Single & $\begin{array}{l}1,705 \\
(17.4)\end{array}$ & $\begin{array}{l}9 \\
(0.5)\end{array}$ & $\begin{array}{l}573 \\
(33.6)\end{array}$ & $\begin{array}{l}935 \\
(54.8)\end{array}$ & $165(9.7)$ & $\begin{array}{l}23 \\
(1.3)\end{array}$ \\
\hline Married & $\begin{array}{l}6,593 \\
(67.3)\end{array}$ & $\begin{array}{l}42 \\
(0.6)\end{array}$ & $\begin{array}{l}1,809 \\
(27.4)\end{array}$ & $\begin{array}{l}3,523 \\
(55.0)\end{array}$ & $\begin{array}{l}1,108 \\
(16.8)\end{array}$ & $\begin{array}{l}109 \\
(1.7)\end{array}$ \\
\hline common-law* & $\begin{array}{l}1,501 \\
(15.3)\end{array}$ & $\begin{array}{l}18 \\
(1.2)\end{array}$ & $424(28.2)$ & $\begin{array}{l}816 \\
(56.1)\end{array}$ & $\begin{array}{l}228 \\
(15.2)\end{array}$ & $\begin{array}{l}15 \\
(1.0)\end{array}$ \\
\hline \multicolumn{7}{|c|}{ Highest level of education $(N=9,797)$} \\
\hline No formal Schooling & $\begin{array}{l}7,661 \\
(78.2)\end{array}$ & $\begin{array}{l}52 \\
(0.7)\end{array}$ & $\begin{array}{l}2,156 \\
(30.1)\end{array}$ & $\begin{array}{l}4,186 \\
(28.1)\end{array}$ & $\begin{array}{l}1163 \\
(15.2)\end{array}$ & $\begin{array}{l}102 \\
(1.3)\end{array}$ \\
\hline $\begin{array}{l}\text { Primary school } \\
\text { completed }\end{array}$ & $975(10.0)$ & $\begin{array}{l}9 \\
(0.9)\end{array}$ & $\begin{array}{l}294 \\
(32.3)\end{array}$ & $\begin{array}{l}516 \\
(30.2)\end{array}$ & $\begin{array}{l}140 \\
(14.4)\end{array}$ & $\begin{array}{l}15 \\
(1.5)\end{array}$ \\
\hline $\begin{array}{l}\text { secondary school } \\
\text { completed }\end{array}$ & $653(6.7)$ & $\begin{array}{l}5 \\
(0.8)\end{array}$ & $\begin{array}{l}190 \\
(32.2)\end{array}$ & $\begin{array}{l}334 \\
(29.1)\end{array}$ & $\begin{array}{l}109 \\
(16.7)\end{array}$ & $\begin{array}{l}15 \\
(2.3)\end{array}$ \\
\hline
\end{tabular}




\section{Number of risk factors}

\begin{tabular}{|c|c|c|c|c|c|c|}
\hline $\begin{array}{l}\text { College/University } \\
\text { completed* }\end{array}$ & $511(5.2)$ & $\begin{array}{l}3 \\
(0.6)\end{array}$ & $\begin{array}{l}166 \\
(40.7)\end{array}$ & $\begin{array}{l}238 \\
(32.5)\end{array}$ & $89(17.4)$ & $\begin{array}{l}615 \\
(2.9)\end{array}$ \\
\hline \multicolumn{7}{|c|}{ Annual income (Birr) $(N=6,636)$} \\
\hline$<=12,000$ Birr & $\begin{array}{l}4,494 \\
(67.7)\end{array}$ & $\begin{array}{l}40 \\
(0.9)\end{array}$ & $\begin{array}{l}1,234 \\
(27.5)\end{array}$ & $\begin{array}{l}2,499 \\
(55.6)\end{array}$ & $\begin{array}{l}662 \\
(14.7)\end{array}$ & $59(1.3$ \\
\hline 12,000 to 23,299 Birr & $\begin{array}{l}1,017 \\
(15.3)\end{array}$ & $\begin{array}{l}2 \\
(0.2)\end{array}$ & $\begin{array}{l}251 \\
(24.7)\end{array}$ & $\begin{array}{l}552 \\
(54.3)\end{array}$ & $\begin{array}{l}192 \\
(18.9)\end{array}$ & $\begin{array}{l}20 \\
(2.0)\end{array}$ \\
\hline $23,300^{+}$Birr & $\begin{array}{l}1,125 \\
(17.0)\end{array}$ & $\begin{array}{l}10 \\
(0.9)\end{array}$ & $\begin{array}{l}324 \\
(28.8)\end{array}$ & $\begin{array}{l}531 \\
(47.2)\end{array}$ & $\begin{array}{l}224 \\
(19.9)\end{array}$ & $\begin{array}{l}36 \\
(3.2)\end{array}$ \\
\hline \multicolumn{7}{|l|}{ Region } \\
\hline Addis Ababa & $814(8.3)$ & $\begin{array}{l}20 \\
(2.5)\end{array}$ & $\begin{array}{l}202 \\
(24.8)\end{array}$ & $\begin{array}{l}381 \\
(46.8)\end{array}$ & $\begin{array}{l}182 \\
(22.4)\end{array}$ & $\begin{array}{l}29 \\
(3.6)\end{array}$ \\
\hline Afar & $384(4)$ & $\begin{array}{l}0 \\
(0.0)\end{array}$ & $84(21.9)$ & $\begin{array}{l}259 \\
(67.4)\end{array}$ & $40(10.4)$ & $1(0.3)$ \\
\hline Amhara & $1,866(19)$ & $\begin{array}{l}1 \\
(0.1)\end{array}$ & $\begin{array}{l}552 \\
(29.6)\end{array}$ & $\begin{array}{l}967 \\
(51.8)\end{array}$ & $\begin{array}{l}337 \\
(18.1)\end{array}$ & $9(0.5)$ \\
\hline B. Gumuz & $383(4)$ & $\begin{array}{l}1 \\
(0.3)\end{array}$ & $81(21.1)$ & $\begin{array}{l}206 \\
(53.8)\end{array}$ & $81(21.1)$ & $\begin{array}{l}14 \\
(3.7)\end{array}$ \\
\hline Dire Dawa & $257(2.6)$ & $\begin{array}{l}0 \\
(0.0)\end{array}$ & $78(30.4)$ & $\begin{array}{l}132 \\
(51.4)\end{array}$ & $43(16.7)$ & $4(1.6)$ \\
\hline Gambela & $295(3)$ & $\begin{array}{l}5 \\
(1.7)\end{array}$ & $\begin{array}{l}111 \\
(37.6)\end{array}$ & $\begin{array}{l}108 \\
(36.6)\end{array}$ & $51(17.3)$ & $\begin{array}{l}20 \\
(6.8)\end{array}$ \\
\hline Harari & $214(2.2)$ & $\begin{array}{l}7 \\
(3.3)\end{array}$ & $95(44.4)$ & $91(42.5)$ & $20(9.3)$ & $1(0.5)$ \\
\hline Oromia & $\begin{array}{l}2,308 \\
(23.6)\end{array}$ & $\begin{array}{l}7 \\
(0.3)\end{array}$ & $\begin{array}{l}599 \\
(26.0)\end{array}$ & $\begin{array}{l}1,341 \\
(58.1)\end{array}$ & $\begin{array}{l}325 \\
(14.1)\end{array}$ & $\begin{array}{l}36 \\
(1.6)\end{array}$ \\
\hline SNNPR & $\begin{array}{l}1,706 \\
(17.4)\end{array}$ & $\begin{array}{l}25 \\
(1.5)\end{array}$ & $\begin{array}{l}561 \\
(32.9)\end{array}$ & $\begin{array}{l}919 \\
(53.9)\end{array}$ & $\begin{array}{l}178 \\
(10.4)\end{array}$ & $\begin{array}{l}23 \\
(1.3)\end{array}$ \\
\hline Somali & $615(6.3)$ & $\begin{array}{l}2 \\
(0.3)\end{array}$ & $\begin{array}{l}266 \\
(43.3)\end{array}$ & $\begin{array}{l}269 \\
(43.7)\end{array}$ & $73(11.9)$ & $5(0.8)$ \\
\hline Tigray & $955(9.7)$ & $\begin{array}{l}1 \\
(0.1)\end{array}$ & $\begin{array}{l}177 \\
(18.5)\end{array}$ & $\begin{array}{l}601 \\
(62.9)\end{array}$ & $\begin{array}{l}171 \\
(17.9)\end{array}$ & $5(0.5)$ \\
\hline \multicolumn{7}{|l|}{ Diabetes $(N=8,788)$} \\
\hline Yes & $243(2.8)$ & $\begin{array}{l}0 \\
(0.0)\end{array}$ & $59(24.3)$ & $\begin{array}{l}139 \\
(57.2)\end{array}$ & $38(15.6)$ & $7(2.9)$ \\
\hline
\end{tabular}

Hypertension $(N=9,675)$ 


\begin{tabular}{|lllllll|}
\hline \multicolumn{7}{|c|}{ Number of risk factors } \\
\hline $\mathrm{SBP}>=140$ or & 1,871 & 15 & 472 & 946 & 387 & 51 \\
$\mathrm{DBP}>=90$ & $(19.3)$ & $(0.8)$ & $(25.2)$ & $(50.6)$ & $(20.7)$ & $(2.7)$ \\
\hline
\end{tabular}

\section{Factors associated with unhealthy lifestyles}

Participants who are male $(\mathrm{AOR}=2.27,95 \% \mathrm{Cl}: 1.95,2.63)$, urban residence $(\mathrm{AOR}=1.76,95 \% \mathrm{Cl}=1.50$, 2.06), older age groups $(30-44 \mathrm{y}: \mathrm{AOR}=1.66,95 \% \mathrm{Cl}=1.38,1.99 ; 45-59 \mathrm{y}: \mathrm{AOR}=1.99,95 \% \mathrm{Cl}=1.60$, $2.47)$ and $60-69$ y $(A O R=1.59,95 \% \mathrm{Cl}=1.182 .15))$; married $(\mathrm{AOR}=1.32,95 \% \mathrm{Cl}=1.04,1.68)$ and earning annual income of more than 12,000 Ethiopian $\operatorname{Birr}(12,000$ to 23,299 : $A O R=1.46,95 \% \mathrm{Cl}=1.20,176$ and $23,300$ and above: $A O R=1.41,95 \% \mathrm{Cl}=1.12,1.75)$ and those with high blood pressure $(\mathrm{AOR}=1.53$, $95 \% \mathrm{Cl}=1.30,1.80)$ had higher odds of unhealthy lifestyle. Participants with higher educational status $(A O R=0.33,95 \% \mathrm{Cl}=0.55,0.97)$ had lower odds of having unhealthy lifestyles (Table 3$)$. 
Table 3

Sociodemographic factors associated with unhealthy lifestyle

\begin{tabular}{|c|c|c|c|c|}
\hline \multirow[t]{2}{*}{ Variables } & $\begin{array}{l}\text { At least two unhealthy } \\
\text { lifestyle risk factors }\end{array}$ & $\begin{array}{l}\text { Three or more unhealthy } \\
\text { lifestyle risk factors }\end{array}$ & $\begin{array}{l}\text { COR } \\
(95 \% \mathrm{Cl})\end{array}$ & $\begin{array}{l}\text { AOR } \\
(95 \% \mathrm{Cl})\end{array}$ \\
\hline & $8533(87.0 \%)$ & $1264(13.0 \%)$ & & \\
\hline \multicolumn{5}{|l|}{ Residence } \\
\hline Rural & $5,864(85.0)$ & $925(15.0)$ & 1 & 1 \\
\hline Urban & $2,285(79.0)$ & $338(21.0)$ & $\begin{array}{l}1.51(1.35 \\
1.68)^{\star \star \star}\end{array}$ & $\begin{array}{l}1.76 \\
(1.50 \\
2.06)\end{array}$ \\
\hline \multicolumn{5}{|l|}{ Sex } \\
\hline Female & $5,110(87.8)$ & $711(12.2)$ & 1 & \\
\hline Male & $3,039(76.4)$ & $937(23.6)$ & $\begin{array}{l}2.22(1.99 \\
2.47)\end{array}$ & $\begin{array}{l}2.27 \\
(1.95 \\
2.63)\end{array}$ \\
\hline \multicolumn{5}{|l|}{ Age (years) } \\
\hline $15-29$ & $3,526(89.1)$ & 431 (10.9) & 1 & 1 \\
\hline $30-44$ & $2,829(80.9)$ & $669(19.1)$ & $\begin{array}{l}1.93(1.70 \\
9.89)^{* * \star}\end{array}$ & $\begin{array}{l}1.66 \\
(1.38 \\
1.99)\end{array}$ \\
\hline $45-59$ & $1,273(75.3)$ & $417(27.7)$ & $\begin{array}{l}2.2 .68 \\
(2.31 \\
12.96)\end{array}$ & $\begin{array}{l}1.99 \\
(1.60 \\
2.47)^{\text {*** }}\end{array}$ \\
\hline $60-69$ & $521(79.9)$ & $131(20.1)$ & $\begin{array}{l}2.06(1.66 \\
6.54)^{\star \star \star}\end{array}$ & $\begin{array}{l}1.59 \\
(1.18 \\
2.15)\end{array}$ \\
\hline \multicolumn{5}{|l|}{ Marital status } \\
\hline Single & 1, $517(89.0)$ & $188(11.0)$ & 1 & 1 \\
\hline Married & $5,374(81.5)$ & $1,217(18.5)$ & $\begin{array}{l}1.83(1.55 \\
2.16)^{\star \star \star}\end{array}$ & $\begin{array}{l}1.32 \\
(1.04 \\
1.68)\end{array}$ \\
\hline common-law & 1,258 (83.8) & $243(16.2)$ & $\begin{array}{l}1.56(1.27 \\
1.91)^{\star \star \star}\end{array}$ & $\begin{array}{l}1.29 \\
(0.94 \\
1.76)\end{array}$ \\
\hline $\begin{array}{l}\text { Highest level of } \\
\text { education }\end{array}$ & & & & \\
\hline
\end{tabular}

COR $=$ Crude odds ratios; $A O R=$ Adjusted odds ratios 


\begin{tabular}{|c|c|c|c|c|}
\hline \multirow[t]{2}{*}{ Variables } & $\begin{array}{l}\text { At least two unhealthy } \\
\text { lifestyle risk factors }\end{array}$ & $\begin{array}{l}\text { Three or more unhealthy } \\
\text { lifestyle risk factors }\end{array}$ & $\begin{array}{l}\text { COR } \\
(95 \% \mathrm{Cl})\end{array}$ & $\begin{array}{l}\text { AOR } \\
(95 \% \mathrm{Cl})\end{array}$ \\
\hline & $8533(87.0 \%)$ & 1264 (13.0\%) & & \\
\hline $\begin{array}{l}\text { No formal } \\
\text { Schooling }\end{array}$ & $6,394(83.5)$ & $1,265(16.5)$ & 1 & 1 \\
\hline $\begin{array}{l}\text { Primary school } \\
\text { completed }\end{array}$ & $819(84.1)$ & $155(15.9)$ & $\begin{array}{l}0.96(0.80 \\
1.14)\end{array}$ & $\begin{array}{l}0.98 \\
(0.77 \\
1.25)\end{array}$ \\
\hline $\begin{array}{l}\text { secondary school } \\
\text { completed }\end{array}$ & $529(81.0)$ & $124(19.0)$ & $\begin{array}{l}1.18(0.96, \\
1.45)\end{array}$ & $\begin{array}{l}0.95(0 . \\
72,1.24)\end{array}$ \\
\hline $\begin{array}{l}\text { College/University } \\
\text { completed* }\end{array}$ & $407(79.6)$ & $104(20.4)$ & $\begin{array}{l}1.29(1.03 \\
1.61)^{*}\end{array}$ & $\begin{array}{l}0.73 \\
(0.55 \\
0.97) \text { * }\end{array}$ \\
\hline \multicolumn{5}{|l|}{$\begin{array}{l}\text { Annual income } \\
\text { (Birr) }\end{array}$} \\
\hline$<=12,000$ & $3,773(84.0)$ & $721(16.0)$ & 1 & 1 \\
\hline 12,000 to 23,299 & $805(79.2)$ & $212(20.8)$ & $\begin{array}{l}1.38(1.16 \\
1.63)^{\star \star \star}\end{array}$ & $\begin{array}{l}1.46 \\
(1.20 \\
1.76)^{* \star \star}\end{array}$ \\
\hline $23,300+$ & $865(76.9)$ & $260(23.1)$ & $\begin{array}{l}1.57(1.34 \\
1.84)^{\star \star \star}\end{array}$ & $\begin{array}{l}1.48 \\
(1.22 \\
1.79)^{* \star *}\end{array}$ \\
\hline \multicolumn{5}{|l|}{ Diabetes } \\
\hline No & $7,122(83.3)$ & $1,423(16.7)$ & 1 & 1 \\
\hline Yes & $198(81.5)$ & 45 (18.5) & $\begin{array}{l}1.14(0.81 \\
1.56)\end{array}$ & $\begin{array}{l}0.70 \\
(0.46 \\
1.04)\end{array}$ \\
\hline \multicolumn{5}{|l|}{$\begin{array}{l}\text { High blood } \\
\text { pressure }\end{array}$} \\
\hline No & $6,605(84.6)$ & $1,199(15.4)$ & 1 & 1 \\
\hline Yes & 1,433 (76.6) & $438(23.4)$ & $\begin{array}{l}1.68(1.49 \\
1.90)^{\star \star \star}\end{array}$ & $\begin{array}{l}1.53 \\
(1.30 \\
1.80)^{\star * \star}\end{array}$ \\
\hline
\end{tabular}

\section{Unhealthy lifestyle factors, diabetes, and hypertension}

Table 4 shows that excessive alcohol intake and overweight/obesity are associated with diabetes and high blood pressure. 
Table 4

Prevalence (\%) and relationships of unhealthy lifestyle factors with diabetes and hypertension in Ethiopia

\begin{tabular}{|c|c|c|c|c|}
\hline \multirow[t]{2}{*}{ Unhealthy lifestyle factors } & \multicolumn{2}{|l|}{ Diabetes } & \multicolumn{2}{|c|}{ High blood pressure } \\
\hline & No & Yes & No & Yes \\
\hline Smoking & \multicolumn{2}{|c|}{$X^{2}=4.48, \mathrm{df}=1, p=0.034$} & \multicolumn{2}{|c|}{$X^{2}=0.14, \mathrm{df}=1, p=0.71$} \\
\hline No & $8,130(92.2)$ & $223(2.5)$ & $7,373(76.2)$ & $1,772(18.3)$ \\
\hline Yes & $441(5.0)$ & $20(0.2)$ & $430(4.4)$ & $99(1.0)$ \\
\hline Excessive alcohol intake & \multicolumn{2}{|c|}{$X^{2}=4.25, \mathrm{df}=1, p=0.04$} & \multicolumn{2}{|c|}{$X^{2}=10.3, d f=1, p=0.001$} \\
\hline No & $6,955(82.4)$ & $207(2.5)$ & $6,409(69.01)$ & $1,454(15.6)$ \\
\hline Yes & $1,259(15.0)$ & $24(0.28)$ & $1,109(11.9)$ & $315(3.4)$ \\
\hline Insufficient physical activity & \multicolumn{2}{|c|}{$X^{2}=0.151, \mathrm{df}=1, p=0.69$} & \multicolumn{2}{|c|}{$\mathrm{X}^{2}=0.02, \mathrm{df}=1, p=0.88$} \\
\hline No & $2,742(33.5)$ & $68(0.8)$ & $2,494(27.7)$ & $572(6.4)$ \\
\hline Yes & $5,250(64.0)$ & $138(1.7)$ & $4,821(53.6)$ & $1,115(12.4)$ \\
\hline Low fruit and vegetable intake & \multicolumn{2}{|c|}{$X^{2}=0.024, \mathrm{df}=1, p=0.88$} & \multicolumn{2}{|c|}{$X^{2}=0.004, \mathrm{df}=1, p=0.9$} \\
\hline No & $153(1.8)$ & $4(0.05)$ & $132(1.4)$ & $32(0.3)$ \\
\hline Yes & $8,308(95.5)$ & 235 & $7,594(79.3)$ & $1,818(19.0)$ \\
\hline Overweight or obesity & \multicolumn{2}{|c|}{$X^{2}=68.24, \mathrm{df}=1, p=<0.00$} & \multicolumn{2}{|c|}{$X^{2}=286.78, \mathrm{df}=1, p=<0.00$} \\
\hline No & $7,431(87.8)$ & $177(2.09)$ & $6879(74.1)$ & $1,464(15.8)$ \\
\hline Yes & 791 (9.3) & $62(0.73)$ & $555(5.98)$ & $383(4.1)$ \\
\hline
\end{tabular}

\section{Discussion}

In this study, we examined the socio-demographic correlates of unhealthy lifestyle factors in Ethiopia, with a focus on smoking, excessive alcohol intake, physical inactivity, low daily fruit and vegetable consumption, and overweight and obesity. A high prevalence of unhealthy lifestyle factors was reported, with $16.7 \%$ having three or more unhealthy lifestyle factors. Unhealthy lifestyles were significantly associated with gender, marital status urban residence, old age, and higher income

Several large, nationally representative surveys such as the Global Burden of Disease study identified that a significant proportion of NCDs and disability-adjusted life years lost across the globe - including in LMICs - are due to mainly modifiable lifestyle factors such as smoking, unhealthy diet, physical inactivity, and inappropriate alcohol consumption (30). In the current study, we found that participants were more likely to engage in two or more unhealthy behaviours. Previous studies $(8,31,32)$ reported 
similar findings. High prevalence and co-occurrence of unhealthy lifestyle factors are in turn associated with high burden of morbidity and premature mortality from chronic illnesses, including cardiovascular diseases, diabetes, chronic obstructive pulmonary disease, and some types of cancer (33). This may eventually lead to healthcare system strain and increased cost of disease management, and productivity loss due to illnesses.

Our findings revealed that a high prevalence of co-occurrence of unhealthy lifestyle factors was significantly associated with place of residence, where participants from urban areas were more likely to have unhealthy lifestyles than their counterparts. This may be because people in urban areas in developing countries, including Ethiopia, are increasingly exposed to unhealthy environments, thence behaviours, compared to rural areas due to economic and social developments. In line with, a relatively sedentary lifestyle, other lifestyle factors, such as smoking, alcohol consumption and overweight and obesity are more rampant (34-37)

Similarly, the current study showed that the likelihood of co-occurrence of risk factors or unhealthy lifestyle increases among participants with higher income, which is in line with previous studies from Ethiopia, Ghana, and Chile. Risk factors, such as overweight and obesity, and low physical activity were associated with wealthier socioeconomic groups (33-35). This is a common occurrence in developing countries' contexts, where people tend to consume energy-dense and high-fat foods and follow a sedentary lifestyle as their economic condition improves $(34,38)$. However, the study showed participants with higher educational status are more prone to engage in physical activity and have a good diet. This finding is similar to a study done in Ghana that; Ghanaian adults were more likely to live a healthier lifestyle with increasing levels of educational attainment (34). This may be because educated people could easily access educational messages on health and risk factors to choose their unhealthy behaviour.

The study also showed variations in the co-existence of unhealthy lifestyle risk factors by sociodemographic factors such as gender, age, and marital status. It was observed that despite mixed findings on gender and the number of unhealthy lifestyles, obesity and insufficient physical activity, were higher among females than males, while males had higher risks for excessive alcohol intake and smoking (35, 39). Likewise, our study showed that older participants were more likely to have an unhealthy lifestyle than younger groups. The current finding is in line with other similar studies that the prevalence of unhealthy lifestyles, such as smoking, excessive alcohol drinking, and obesity, increased with age (38, 39). In the study, those who were married also showed a significant association with higher odds of an unhealthy lifestyle. In Ethiopia or other developing countries, evidence indicates that married people are more likely to adopt a sedentary lifestyle $(38,40,41)$.

Overall, the study shows that the co-occurrence of unhealthy lifestyle factors is alarmingly high. Individual-level risk factors such as excessive alcohol intake and overweight and obesity were found to be associated with diabetes and high blood pressure. There is a need to emphasize providing continuous and focused health promotion and behavioural support to adopt healthy lifestyle habits. 


\section{Implication for policy and practice}

Consistent with the rapid socio-economic development and lifestyle changes Ethiopia has seen over the last few decades, there has been a stark epidemiological transition from one that is predominantly communicable disease towards the double burden of communicable and non-communicable diseases. While several studies in Ethiopia have documented the prevalence of unhealthy lifestyle behaviours individually, our study is the first to document the co-occurrence of these risk factors according to sociodemographic characteristics. Findings from this study can be used by policymakers as an input to implement comprehensive, policy-level behavioural and public health interventions that will ultimately reduce the burden of NCDs in Ethiopia through a healthy lifestyle program.

Furthermore, understanding the increasing risk of NCDs due to the co-occurrence of multiple risk factors will also help to design comprehensive and coordinated interventions that will reduce the burden on the already overstretched healthcare system. Our findings also call for designing context-specific and tailored interventions in addressing the risk factors for NCDs. Risk factors, such as age, sex, and others must be taken into consideration when designing healthy lifestyle programs.

This study has both strengths and limitations. The main strengths are that: (1) it is the first study that assesses the co-occurrence NCD risk factors, and (2) the study was performed nationally with relatively large sample size. The limitations are: (1) most of the study participants are from rural areas, (2) we used the 2015 survey, and the data may not reflect the current unhealthy lifestyle factors.

\section{Conclusion}

The present study showed one in eight participants had three or more unhealthy lifestyle factors and the distributions were significantly different by sociodemographic characteristics. Participants with higher educational status had lower odds of an unhealthy lifestyle.

\section{Declarations}

\section{Data availability statement}

The study data are available upon request from the corresponding author through yalassefa@gmail.com

\section{Acknowledgment}

The authors acknowledge the Ethiopian Public Health Institute (EPHI) for providing the data, Australian Based Ethiopian Researcher Network (ABReN) for organizing this research team and facilitating the data request, and the Curtin University for ethical approval. 


\section{Authors contribution}

YAG, DNK, KAA, KYA, and YAM contributed to conceiving the research idea and design. YAG design and conduct the analysis. DNK is involved in data cleaning. YAG, YAM, DAE, HGT, and ATG drafted the manuscript. DNK, KAA, YA, and BMZ reviewed and provided extensive feedback on the manuscript. All authors critically reviewed, edited, and approved the final manuscript for submission.

\section{References}

1. World Health Organization. Global Health Estimates 2016: Deaths by Cause, Age, Sex, by Country and by Region, 2000-2016.. Geneva: World Health Organization; 2018. Report No.: 9241514620.

2. World Health Organization. Global status report on noncommunicable diseases 2014. World Health Organization; 2014. Report No.: 9241564857 Contract No.: WHO/NMH/NVI/15.1.

3. World Health Organization. Noncommunicable diseases country profiles 2018. 2018. Report No.: 9241514620.

4. Bloom DE, Chisholm D, Jané-Llopis E, Prettner K, Stein A, Feigl A. From Burden to "Best Buys": Reducing the Economic Impact of Non-Communicable Diseases. World Health Org, Geneva, Switzerland. 2011.

5. Bloom DE, Cafiero E, Jané-Llopis E, Abrahams-Gessel S, Bloom LR, Fathima S, et al. The global economic burden of noncommunicable diseases. Program on the Global Demography of Aging; 2012.

6. Bollyky TJ, Templin T, Cohen M, Dieleman JL. Lower-income countries that face the most rapid shift in noncommunicable disease burden are also the least prepared. Health Affairs. 2017;36(11):186675 .

7. World Health Organization. WHO global coordination mechanism on the prevention and control of non-communicable diseases: progress report 2014-2016. Geneva: WHO; 2017.

8. GBD 2015 Risk Factors Collaborators. Global, regional, and national comparative risk assessment of 79 behavioural, environmental and occupational, and metabolic risks or clusters of risks, 19902015: a systematic analysis for the Global Burden of Disease Study 2015. Lancet. 2016;388(10053):1659-724.

9. Wang H, Naghavi M, Allen C, Barber RM, Bhutta ZA, Carter A, et al. Global, regional, and national life expectancy, all-cause mortality, and cause-specific mortality for 249 causes of death, 1980-2015: a systematic analysis for the Global Burden of Disease Study 2015. The lancet. 2016;388(10053):1459-544.

10. The Federal Democratic Republic of Ethiopia Ministry of Health. Health sector transformation plan (2015/16-2019/20). Federal Ministry of Health Addis Ababa, Ethiopia; 2015.

11. Misganaw A, Haregu TN, Deribe K, Tessema GA, Deribew A, Melaku YA, et al. National mortality burden due to communicable, non-communicable, and other diseases in Ethiopia, 1990-2015: 
findings from the Global Burden of Disease Study 2015. Population health metrics. 2017;15(1):29.

12. Ford ES, Bergmann MM, Kröger J, Schienkiewitz A, Weikert C, Boeing H. Healthy living is the best revenge: findings from the European Prospective Investigation Into Cancer and Nutrition-Potsdam study. Archives of internal medicine. 2009;169(15):1355-62.

13. Nugent R, Bertram MY, Jan S, Niessen LW, Sassi F, Jamison DT, et al. Investing in non-communicable disease prevention and management to advance the Sustainable Development Goals. The Lancet. 2018;391(10134):2029-35.

14. Yang Z-Y, Yang Z, Zhu L, Qiu C. Human behaviors determine health: strategic thoughts on the prevention of chronic non-communicable diseases in China. International journal of behavioral medicine. 2011;18(4):295-301.

15. Poortinga W. The prevalence and clustering of four major lifestyle risk factors in an English adult population. Preventive medicine. 2007;44(2):124-8.

16. Al-Hanawi MK, Keetile M. Socio-economic and demographic correlates of non-communicable disease risk factors among adults in Saudi Arabia. Frontiers in Medicine. 2021;8.

17. El Ghardallou M, Maatoug J, Harrabi I, Fredj SB, Jihene S, Dendana E, et al. Socio-demographic association of non communicable diseases' risk factors in a representative population of school children: A cross-sectional study in Sousse (Tunisia). International journal of adolescent medicine and health. 2017;29(5).

18. Ryu SY, Park J, Choi SW, Han MA. Associations between socio-demographic characteristics and healthy lifestyles in Korean Adults: the result of the 2010 Community Health Survey. Journal of Preventive Medicine and Public Health. 2014;47(2):113.

19. Lv J, Liu Q, Ren Y, Gong T, Wang S, Li L, et al. Socio-demographic association of multiple modifiable lifestyle risk factors and their clustering in a representative urban population of adults: a crosssectional study in Hangzhou, China. International Journal of Behavioral Nutrition and Physical Activity. 2011;8(1):40.

20. World Health Organization. Best buys' and other recommended interventions for the prevention and control of noncommunicable diseases. Geneva: World Health Organization. 2017.

21. World Health Organization. WHO STEPS surveillance manual: the WHO STEPwise approach to chronic disease risk factor surveillance. World Health Organization; 2005. Report No.: 9241593830.

22. Ethiopian Public Health Institute (EPHI). Ethiopia Steps Report on Risk Factors for NonCommunicable Disease and Prevalence of Selected NCDs. Addis Ababa: EPHI; 2016.

23. Li Y, Pan A, Wang DD, Liu X, Dhana K, Franco OH, et al. Impact of healthy lifestyle factors on life expectancies in the US population. Circulation. 2018;138(4):345-55.

24. World Health Organization. Guidelines on physical activity and sedentary behaviour. Geneva: World Health Organization; 2020.

25. NIAAA A. Understanding the Impact of Alcohol on Human Health and Well-Being. Bethesda, MD, USA: NIAAA; 2017. 
26. Agudo A, Joint F. Measuring intake of fruit and vegetables [electronic resource]: World Health Organization; 2005.

27. Report of a Joint WHO/FAO Expert Consultation. Diet, nutrition and the prevention of chronic diseases. World Health Organ Tech Rep Ser. 2003;916(i-viii):1-149.

28. Hirvonen K. Rural-urban differences in children's dietary diversity in Ethiopia: A Poisson decomposition analysis. Economics Letters. 2016;147:12-5.

29. Vandenbroucke JP, von Elm E, Altman DG, Gøtzsche PC, Mulrow CD, Pocock SJ, et al. Strengthening the Reporting of Observational Studies in Epidemiology (STROBE): Explanation and elaboration. International Journal of Surgery. 2014;12(12):1500-24.

30. Roth GA, Mensah GA, Johnson CO, Addolorato G, Ammirati E, Baddour LM, et al. Global Burden of Cardiovascular Diseases and Risk Factors, 1990-2019: Update From the GBD 2019 Study. Journal of the American College of Cardiology. 2020;76(25):2982-3021.

31. Krokstad S, Ding D, Grunseit AC, Sund ER, Holmen TL, Rangul V, et al. Multiple lifestyle behaviours and mortality, findings from a large population-based Norwegian cohort study - The HUNT Study. BMC Public Health. 2017;17(1):58.

32. Rizzuto D, Fratiglioni L. Lifestyle factors related to mortality and survival: a mini-review. Gerontology. 2014;60(4):327-35.

33. Al-Maskari F. Lifestyle diseases: An economic burden on the health services. UN Chronicle The Magazine of the United Nations. 2010.

34. Tagoe HA, Dake FAA. Healthy lifestyle behaviour among Ghanaian adults in the phase of a health policy change. Globalization and Health. 2011;7(1):7.

35. Federal Democratic Republic of Ethiopia MoH. The Ethiopia Noncommunicable Diseases and Injuries (NCDI) Commission Report Summary. Addressing the Impact of Noncommunicable Diseases and Injuries in Ethiopia. 2018.

36. Kingue S, Ngoe CN, Menanga AP, Jingi AM, Noubiap JJN, Fesuh B, et al. Prevalence and risk factors of hypertension in urban areas of Cameroon: a nationwide population-based cross-sectional study. The Journal of Clinical Hypertension. 2015;17(10):819-24.

37. Rawal LB, Biswas T, Khandker NN, Saha SR, Bidat Chowdhury MM, Khan ANS, et al. Noncommunicable disease (NCD) risk factors and diabetes among adults living in slum areas of Dhaka, Bangladesh. PloS one. 2017;12(10):e0184967.

38. Abrha S, Shiferaw S, Ahmed KY. Overweight and obesity and its socio-demographic correlates among urban Ethiopian women: evidence from the 2011 EDHS. BMC Public Health. 2016;16(1):636.

39. Peer N, Bradshaw D, Laubscher R, Steyn N, Steyn K. Urban-rural and gender differences in tobacco and alcohol use, diet and physical activity among young black South Africans between 1998 and 2003. Global Health Action. 2013;6(1):19216.

40. Gichu M, Asiki G, Juma P, Kibachio J, Kyobutungi C, Ogola E. Prevalence and predictors of physical inactivity levels among Kenyan adults (18-69 years): an analysis of STEPS survey 2015. BMC Public Health. 2018;18(3):1217. 
41. Cavazzotto TG, de Lima Stavinski NG, Queiroga MR, da Silva MP, Cyrino ES, Serassuelo Junior H, et al. Age and Sex-Related Associations between Marital Status, Physical Activity and TV Time. Int J Environ Res Public Health. 2022;19(1).

\section{Figures}

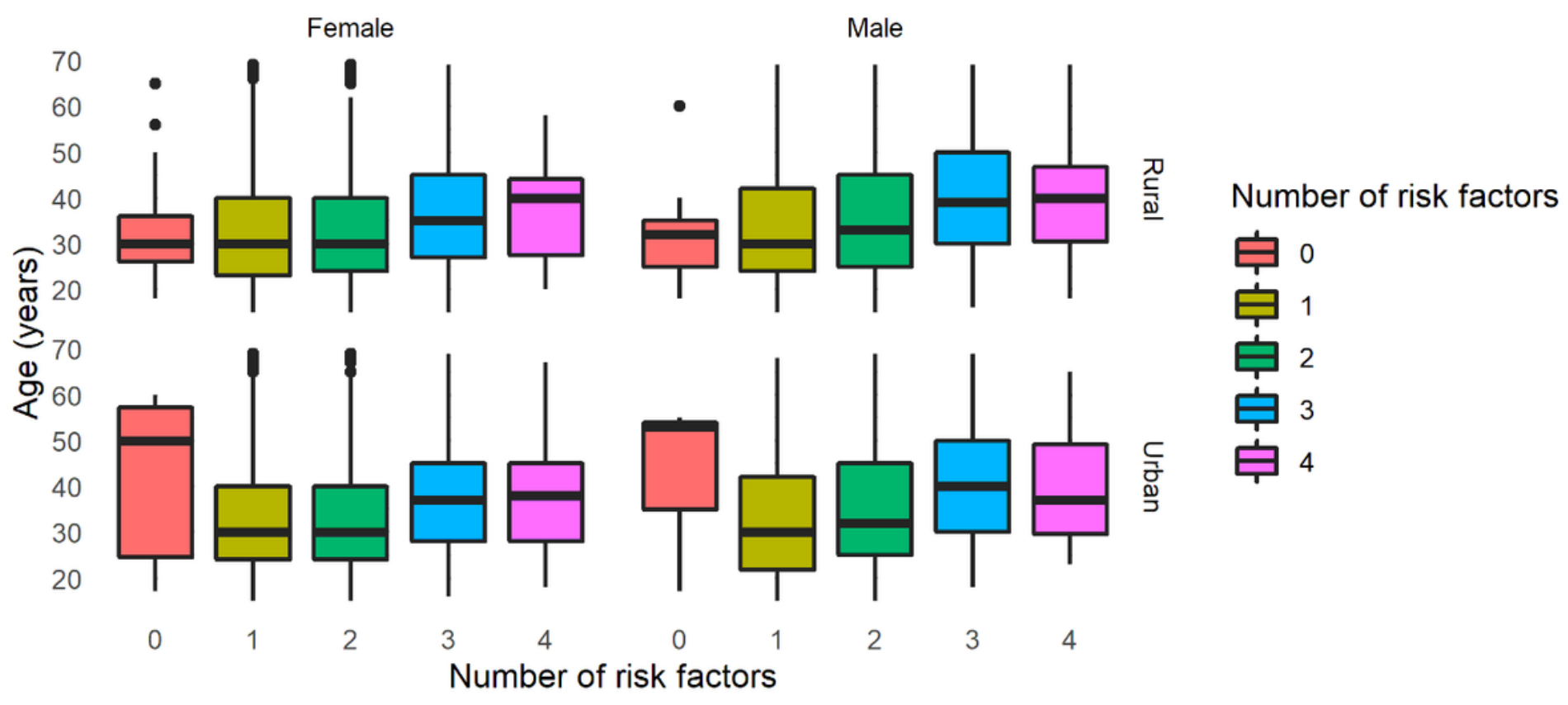

Figure 1

Distributions of unhealthy lifestyle scores across age by sex and residence of the study participants in Ethiopia 


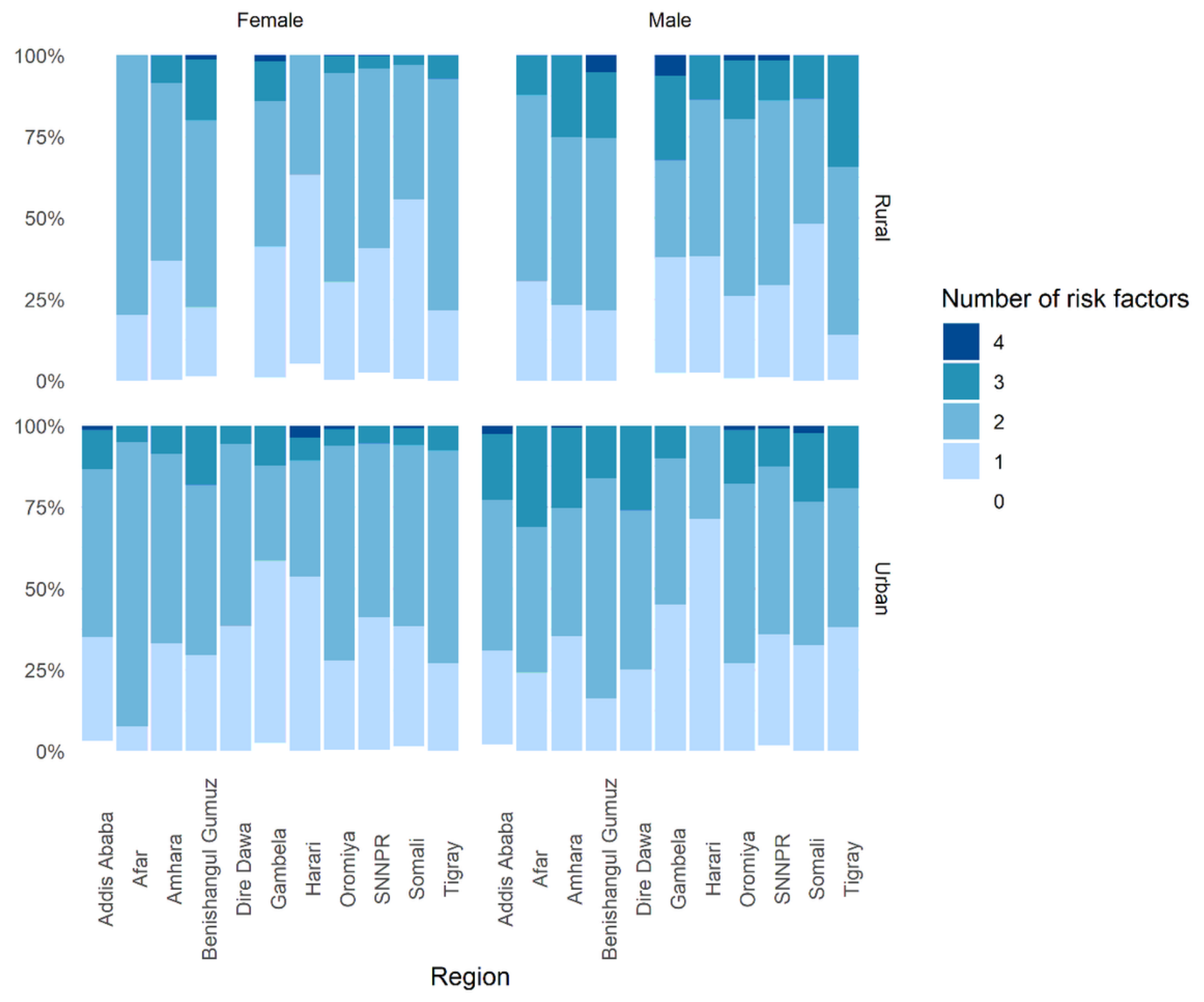

Figure 2

Distributions of unhealthy lifestyle scores of participants in each region by sex and residence of the study participants in Ethiopia 